\title{
Design, synthesis, characterization and antitubercular activity of some 2-heterocycle-substituted phenothiazines
}

\author{
Amit R.Trivedi, Arif B. Siddiqui, and Viresh H. Shah* \\ Chemical Research Laboratory, Department of Chemistry, Saurashtra University, \\ Rajkot-360005, India \\ E-mail:drvireshshah@gmail.com
}

\begin{abstract}
Some novel 2-heterocycle-substituted phenothiazines having a pyrazolo $[3,4-d]$ pyrimidine nucleus have been synthesized by using the Biginelli multi-component cyclocondensation reaction. The products were characterized by FT-IR, ${ }^{1} \mathrm{H}$ NMR, ${ }^{13} \mathrm{C}$ NMR, mass spectra and elemental analysis. The products were evaluated for their antitubercular activity against Mycobacterium tuberculosis $\mathrm{H}_{37} \mathrm{Rv}$.
\end{abstract}

Keywords: Phenothiazine, cyclocondensation, pyrazolo[3,4-d]pyrimidine

\section{Introduction}

The chemistry of nitrogen-sulfur heteroatom containing aromatic compounds is becoming more popular as an area of research. Phenothiazines and related compounds have shown diverse biological activities including as tranquilizers, ${ }^{1}$ anti-inflammatory, ${ }^{2}$ antimalarial, ${ }^{3}$ antipsychotropic, ${ }^{4}$ antimicrobial, ${ }^{5}$ antitubercular, ${ }^{6,7}$ antitumour ${ }^{8-10}$ and stimulation of the penetration of anticancer agents via the blood-brain barrier. They bind to physiological targets or receptors, producing many possible mechanisms of actions. However, solid cancers of the brain and stomach are generally resistant to chemotherapeutic agents. ${ }^{11}$ Phenothiazines are inexpensive and widely available, and therefore have been examined as anticancer drugs.

A slight variation in the substitution pattern on the phenothiazine nucleus often causes a marked difference in activities and therefore phenothiazines with various substituents are being synthesized and tested for activities in search of better medicinal agents. It has been reported ${ }^{12}$ that some phenothiazines inhibit intracellular replication of viruses including human immunodeficiency viruses (HIV). Furthermore, some of these derivatives have been reported to exhibit significant anticancer activities ${ }^{13,14}$ and great interest has arisen in the design and synthesis of new phenothiazines to explore their anticancer activities. The pyrimidine nucleus, 
which has a useful structure for further molecular exploration for the development of new derivatives with different biological activities, has received much attention in recent years. ${ }^{15}$

Pyrimidine derivatives are of interest because of their pharmacological properties ${ }^{15-26}$ including antiviral, ${ }^{16}$ antitumour, ${ }^{19}$ antibacterial ${ }^{20-24}$ and antihypertensive ${ }^{18}$ effects. Several synthetic strategies have been reported for the preparation of pyrimidine derivatives. ${ }^{24,27-33}$ Most of these are based on modification of the classical one-pot Biginelli reaction ${ }^{24,28-32}$ and in some cases on more complex multi-step processes, ${ }^{33,34}$ which may involve the use of some expensive and commercially non-available materials. Owing to the versatility of pyrimidines and as a continuation of our previous work, ${ }^{35}$ we have extended the convenient Biginelli reaction to include some pyrimidine derivatives containing a phenothiazine nucleus.

\section{Results and Discussion}

\section{Chemistry}

The classical three-component Biginelli condensation is usually carried out in alcoholic solution containing a few drops of concentrated hydrochloric or sulfuric acid as catalyst, although other systems such as $\mathrm{THF} / \mathrm{HCl}$, dioxane $/ \mathrm{HCl}$, or acetic acid/ $\mathrm{HCl}$ have also been employed. ${ }^{36}$ One major drawback of the classical Biginelli protocol is the low yield that is frequently encountered when using sterically more demanding aldehydes or 1,3-dicarbonyl compounds. ${ }^{36}$

In order to promote conditions that would favor higher yields of products, we have recently performed Biginelli condensations using different catalysts such as $\mathrm{PPA}, \mathrm{AlCl}_{3}, \mathrm{BF}_{3}$ etc. We found that using phosphorus pentoxide as a catalyst in the Biginelli one-pot protocol, gave a significant increase in the yields of DHPMs, especially for systems that give only moderate yields using traditional Biginelli conditions.

Several pyrimidine derivatives containing a phenothiazine nucleus were synthesized at reflux temperature. Reaction of 5-methyl-2-(10H-phenothiazin-2-yl)-2,4-dihydro-3H-pyrazol-3-one (2), an appropriate aldehyde, urea and phosphorus pentoxide under reflux conditions afforded 3methyl-1-(10H-phenothiazin-2-yl)-4-phenyl-6-hydroxy-4,5-dihydro- $1 H$-pyrazolo[3,4- $d$ ]pyrimidines (3a-h) (Scheme 1). The yields of the products were found to be excellent (80-90\%). The structures of the synthesized compounds were assigned on the basis of IR, ${ }^{1} \mathrm{H}$ NMR spectra, ${ }^{13} \mathrm{C}$ NMR, mass spectra and purity proven by elemental analysis. In the ${ }^{1} \mathrm{H}$ NMR spectra of (3a-h) a sharp peak representing the methine proton of the pyrimidine was observed in the range of 5.12$5.28 \delta$ confirming the formation of the pyrazolo $[3,4-d]$ pyrimidine nucleus. 
<smiles>CSc1ccc2c(c1)Nc1ccccc1S2</smiles><smiles>NNc1ccc2c(c1)Nc1ccccc1S2</smiles>

(1)<smiles>CC1=NN(c2ccc3c(c2)Nc2ccccc2S3)C(=O)C1</smiles>

(2)

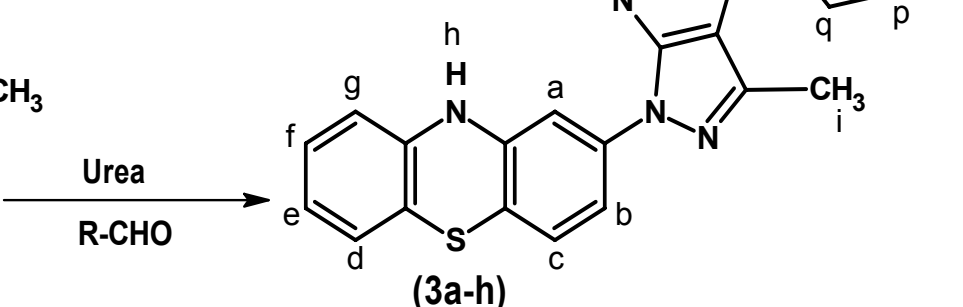

\begin{tabular}{lllllll}
\hline Sr. No. & $\mathbf{R}$ & $\mathbf{m}$ & $\mathbf{n}$ & $\mathbf{0}$ & $\mathbf{p}$ & $\mathbf{q}$ \\
\hline $\mathbf{3 a}$ & $\mathrm{H}$ & $\mathrm{H}$ & $\mathrm{H}$ & $\mathrm{H}$ & $\mathrm{H}$ & $\mathrm{H}$ \\
$\mathbf{3 b}$ & $2-\mathrm{OH}$ & $\mathrm{OH}$ & $\mathrm{H}$ & $\mathrm{H}$ & $\mathrm{H}$ & $\mathrm{H}$ \\
$\mathbf{3 c}$ & $4-\mathrm{OH}$ & $\mathrm{H}$ & $\mathrm{H}$ & $\mathrm{OH}$ & $\mathrm{H}$ & $\mathrm{H}$ \\
$\mathbf{3 d}$ & $2-\mathrm{Cl}$ & $\mathrm{Cl}$ & $\mathrm{H}$ & $\mathrm{H}$ & $\mathrm{H}$ & $\mathrm{H}$ \\
$\mathbf{3 e}$ & $4-\mathrm{Cl}$ & $\mathrm{H}$ & $\mathrm{H}$ & $\mathrm{Cl}$ & $\mathrm{H}$ & $\mathrm{H}$ \\
$\mathbf{3 f}$ & $2-\mathrm{NO}_{2}$ & $\mathrm{NO}_{2}$ & $\mathrm{H}$ & $\mathrm{H}$ & $\mathrm{H}$ & $\mathrm{H}$ \\
$\mathbf{3 g}$ & $3-\mathrm{NO}_{2}$ & $\mathrm{H}$ & $\mathrm{NO}_{2}$ & $\mathrm{H}$ & $\mathrm{H}$ & $\mathrm{H}$ \\
$\mathbf{3 h}$ & $4-\mathrm{OCH}_{3}$ & $\mathrm{H}$ & $\mathrm{H}$ & $\mathrm{OCH}_{3}$ & $\mathrm{H}$ & $\mathrm{H}$ \\
\hline
\end{tabular}

Scheme 1

\section{Antitubercular activity}

The antitubercular activity of the compounds was assessed at the Tuberculosis Antimicrobial Acquisition and Co-ordination Facility (TAACF), U.S.A. Primary screening of the compounds was conducted at $>6.25 \mu \mathrm{g} / \mathrm{ml}$ against Mycobacterium tuberculosis $\mathrm{H}_{37} \mathrm{Rv}$ in BECTEC 12B medium using the BACTEC 460 radiometric system. The antitubercular activities are represented in Table 1. 
Table 1. Antitubercular activity of $\mathbf{3 a - h}$

\begin{tabular}{cllccc}
\hline Sr. No. & $\mathrm{R}$ & Molecular formula & MIC & \% Inh & Activity \\
\hline 3a & $\mathrm{H}$ & $\mathrm{C}_{24} \mathrm{H}_{20} \mathrm{~N}_{6} \mathrm{~S}$ & $>6.25$ & 79 & - \\
3b & $2-\mathrm{OH}$ & $\mathrm{C}_{24} \mathrm{H}_{20} \mathrm{~N}_{6} \mathrm{OS}$ & $>6.25$ & 75 & - \\
3c & $4-\mathrm{OH}$ & $\mathrm{C}_{24} \mathrm{H}_{20} \mathrm{~N}_{6} \mathrm{OS}$ & $<6.25$ & 94 & + \\
3d & $2-\mathrm{Cl}$ & $\mathrm{C}_{24} \mathrm{H}_{20} \mathrm{~N}_{6} \mathrm{SCl}$ & $<6.25$ & 92 & + \\
3e & $4-\mathrm{CI}$ & $\mathrm{C}_{24} \mathrm{H}_{20} \mathrm{~N}_{6} \mathrm{SCl}$ & $<6.25$ & 94 & + \\
3f & $2-\mathrm{NO}_{2}$ & $\mathrm{C}_{24} \mathrm{H}_{19} \mathrm{~N}_{7} \mathrm{O}_{2} \mathrm{~S}$ & $>6.25$ & 74 & - \\
3g & $3-\mathrm{NO}_{2}$ & $\mathrm{C}_{24} \mathrm{H}_{19} \mathrm{~N}_{7} \mathrm{O}_{2} \mathrm{~S}$ & $>6.25$ & 77 & - \\
3h & $4-\mathrm{OCH}_{3}$ & $\mathrm{C}_{25} \mathrm{H}_{22} \mathrm{~N}_{6} \mathrm{OS}$ & $>6.25$ & 63 & - \\
\hline
\end{tabular}

By visualizing the antitubercular data, it could be observed that all the compounds displayed mild to moderate activity. Compounds $\mathbf{3} \mathbf{c}, \mathbf{3 d}$ and $\mathbf{3 e}$ were found to be particularly active against Mycobacterium tuberculosis $\mathrm{H}_{37} \mathrm{Rv}$ strain.

\section{Conclusions}

In conclusion, we have developed a simple and efficient method for the synthesis of pyrimidines having a phenothiazine nucleus. We also believe that the procedural simplicity, the efficiency and the easy accessibility of the reaction partners gives access to a wide array of heterocyclic frameworks equipped with a pendant phenothiazine unit. Other compounds of this group are presently under investigation.

\section{Experimental Section}

General Procedures. All chemicals were purchased from Aldrich Chemicals (Mumbai, India) and were used without further purification. Melting points were determined in open capillary tubes and are uncorrected. Formation of the compounds was routinely checked by TLC using Silica $\mathrm{G}$ and the spots were exposed to iodine vapour for visualization. ${ }^{1} \mathrm{H}$ NMR spectra were obtained in $\mathrm{CDCl}_{3}$ solution on a Bruker DPX $300 \mathrm{MHz}$ spectrometer. ${ }^{13} \mathrm{C}-\mathrm{NMR}$ (75 and 125 MHz) spectra were measured on a Bruker AC 200, DPX 300 and ARX 500, at $25{ }^{\circ} \mathrm{C}$, in $\mathrm{CDCl}_{3}$. IR spectra were recorded on a Shimadzu 8400 spectrometer in $\operatorname{KBr}\left(\gamma\right.$ in $\left.\mathrm{cm}^{-1}\right)$. Elemental analyses of the newly synthesized compounds were carried out on Carlo Erba 1108 analyzer.

Synthesis of 2-hydrazinophenothiazine (1). A mixture of 2-methylthiophenothiazine $2.45 \mathrm{gm}$ $(0.01 \mathrm{~mol})$ and hydrazine hydrate $(10 \mathrm{ml})$ was refluxed for $8 \mathrm{~h}$. The reaction mixture was poured in to ice cold water; the crude product was filtered, dried and recrystallized from $95 \%$ ethanol. Yield 82\%, mp. 122-124 ${ }^{\circ} \mathrm{C}$. IR (KBr): 3335 (NH), 653 (C-S-C). ${ }^{1} \mathrm{H}$ NMR (300 MHz, $\left.\mathrm{CDCl}_{3}\right): \delta$ 
7.45-7.76 (m, 7H, Ar-H), $9.15(\mathrm{~s}, 1 \mathrm{H}, \mathrm{NH}), 7.86-7.95\left(\mathrm{~m}, 3 \mathrm{H}, \mathrm{NHNH}_{2}\right) ;{ }^{13} \mathrm{C}-\mathrm{NMR}\left(\mathrm{CDCl}_{3}\right): \delta$ $103,103.5,107,114.4,116.8,122.3,127.2,128.3,142.3,143,150.6$. Mass $(\mathrm{m} / \mathrm{z}): 229$. Anal. (\%) for $\mathrm{C}_{12} \mathrm{H}_{11} \mathrm{~N}_{3} \mathrm{~S}$, Calcd. C, 62.86; H, 4.84; N, 18.33. Found: C, 62.82; H, 4.80; N, 18.30 .

Synthesis of 5-methyl-2-(10H-phenothiazin-2-yl)-2,4-dihydro-3H-pyrazol-3-one (2). A mixture of 2-hydrazinophenothiazine $2.29 \mathrm{~g}(0.01 \mathrm{~mol})$ and ethyl acetoacetate $1.3 \mathrm{ml}(0.01 \mathrm{~mol})$ in $30 \% \mathrm{w} / \mathrm{w}$ sodium ethoxide $(20 \mathrm{ml})$ was heated under reflux for $12 \mathrm{~h}$. The reaction mixture was poured into ice cold water; the crude product was filtered, dried and recrystallized from $95 \%$ ethanol. Yield 68\%, mp. 111-113 ${ }^{\circ} \mathrm{C}$. IR (KBr): $3330(\mathrm{NH}), 650$ (C-S-C). ${ }^{1} \mathrm{H}$ NMR (300 MHz, $\left.\mathrm{CDCl}_{3}\right): \delta 2.47\left(\mathrm{~s}, 3 \mathrm{H}, \mathrm{CH}_{3}\right), 4.26\left(\mathrm{~s}, 2 \mathrm{H}, \mathrm{CH}_{2}\right), 7.66-7.90(\mathrm{~m}, 7 \mathrm{H}, \mathrm{Ar}-\mathrm{H}), 9.03(\mathrm{~s}, 1 \mathrm{H}, \mathrm{NH}) ;{ }^{13} \mathrm{C}-$ NMR $\left(\mathrm{CDCl}_{3}\right): \delta 16.7,42.4,111.3,111.8,112.1,114.4,116.4,122.3,127.1,128.2,139.6,142.5$, 162.7, 174.1. Mass (m/z): 229. Anal. (\%) for $\mathrm{C}_{12} \mathrm{H}_{11} \mathrm{~N}_{3} \mathrm{~S}$, Calcd. C, 62.86; H, 4.84; N, 18.33 . Found: C, 62.82; H, 4.80; N, 18.30 .

General procedure for synthesis of compounds 3a-h. 3-Methyl-1-(10H-phenothiazin-2-yl)4-phenyl-6-hydroxy-4,5-dihydro-1 $H$-pyrazolo[3,4-d]pyrimidine (3a)

A mixture of benzaldehyde $1.06 \mathrm{ml}(0.01 \mathrm{~mol})$, 5-methyl-2-(10H-phenothiazin-2-yl)-2,4dihydro-3H-pyrazol-3-one $2.95 \mathrm{gm}(0.01 \mathrm{~mol})$, urea $0.76 \mathrm{gm}(0.01 \mathrm{~mol})$ and phosphorus pentoxide $(200 \mathrm{mg})$ in $95 \%$ ethanol $(30 \mathrm{ml})$ was heated under refluxed condition for 5 hours. After cooling to rt., the crystalline product was filtered and recrystallized from ethanol. Yield 74\%, mp. 181-183 ${ }^{\circ} \mathrm{C}$. IR (KBr): $3330(\mathrm{NH}), 1615(\mathrm{C}=\mathrm{N}), 1642(\mathrm{C}-\mathrm{N}), 651$ (C-S-C). ${ }^{1} \mathrm{H}$ NMR $\left(300 \mathrm{MHz}, \mathrm{CDCl}_{3}\right): \delta 2.42\left(\mathrm{~S}, 3 \mathrm{H}, \mathrm{H}_{\mathrm{i}}\right), 5.12\left(\mathrm{~S}, 1 \mathrm{H}, \mathrm{H}_{1}\right), 7.42-7.68\left(\mathrm{~m}, 12 \mathrm{H}, \mathrm{H}_{\mathrm{a}-\mathrm{g}, \mathrm{m}-\mathrm{q}}\right), 8.42(\mathrm{~s}$, $\left.1 \mathrm{H}, \mathrm{H}_{\mathrm{h}}\right), 9.11\left(\mathrm{~s}, 1 \mathrm{H}, \mathrm{H}_{\mathrm{k}}\right) ;{ }^{13} \mathrm{C}-\mathrm{NMR}\left(\mathrm{CDCl}_{3}\right): \delta 26.3,41.1,52.3,60.1,103.1,103.7,104.9,114.2$, 116.7, 122.1, 126, 127.1, 127.7, 128.1, 128.9, 136.1, 142.1, 143.3, 155.1, 164. Mass (m/z): 425. Anal. (\%) for $\mathrm{C}_{24} \mathrm{H}_{19} \mathrm{~N}_{5} \mathrm{OS}$, Calcd. C, 67.74; H, 4.50; N, 16.46. Found: C, 67.70; H, 4.47; N, 16.41 .

3-Methyl-1-(10H-phenothiazin-2-yl)-4-(2-hydroxyphenyl)-6-hydroxy-4,5-dihyd-ro-1Hpyrazolo[3,4-d]pyrimidine (3b). Yield 79\%, mp. 150-152 ${ }^{\circ} \mathrm{C}, \mathrm{IR}(\mathrm{KBr}): 3333(\mathrm{NH}), 1613(\mathrm{C}=$ $\mathrm{N}), 1640(\mathrm{C}-\mathrm{N}), 655(\mathrm{C}-\mathrm{S}-\mathrm{C}) .{ }^{1} \mathrm{H}$ NMR $\left(300 \mathrm{MHz}, \mathrm{CDCl}_{3}\right): \delta 2.40\left(\mathrm{~s}, 3 \mathrm{H}, \mathrm{H}_{\mathrm{i}}\right), 5.20\left(\mathrm{~s}, 1 \mathrm{H}, \mathrm{H}_{1}\right)$, $7.62-8.12\left(\mathrm{~m}, 11 \mathrm{H}, \mathrm{H}_{\mathrm{a}-\mathrm{g}, \mathrm{n}-\mathrm{q}}\right), 8.35\left(\mathrm{~s}, 1 \mathrm{H}, \mathrm{H}_{\mathrm{h}}\right), 9.15\left(\mathrm{~s}, 1 \mathrm{H}, \mathrm{H}_{\mathrm{k}}\right), 10.08\left(\mathrm{~s}, 1 \mathrm{H}, \mathrm{H}_{\mathrm{m}}\right) ;{ }^{13} \mathrm{C}-\mathrm{NMR}$ $\left(\mathrm{CDCl}_{3}\right): \delta 26.4,34.4,52.8,60,103.2,103.7,105.1,114.4,115.7,116.6,121.3,122.3,127.1$, 127.4, 127.7, 128.3, 142.3, 143.1, 155.1, 155.7, 162.9. Mass $(\mathrm{m} / \mathrm{z})$ : 441. Anal. (\%) for $\mathrm{C}_{24} \mathrm{H}_{19} \mathrm{~N}_{5} \mathrm{O}_{2} \mathrm{~S}$, Calcd. C, 65.29; H, 4.34; N, 15.86; Found: C, 65.25; H, 4.38; N, 15.83.

3-Methyl-1-(10H-phenothiazin-2-yl)-4-(4-hydroxyphenyl)-6-hydroxy-4,5-dihydro-1H-pyrazolo[3,4-d]pyrimidine (3c).Yield 81\%, mp. 169-171 ${ }^{\circ} \mathrm{C}$, IR $(\mathrm{KBr}): 3328(\mathrm{NH}), 1612(\mathrm{C}=\mathrm{N})$, $1642(\mathrm{C}-\mathrm{N}), 645(\mathrm{C}-\mathrm{S}-\mathrm{C}) .{ }^{1} \mathrm{H}$ NMR $\left(300 \mathrm{MHz}, \mathrm{CDCl}_{3}\right): \delta 2.44\left(\mathrm{~s}, 3 \mathrm{H}, \mathrm{H}_{\mathrm{i}}\right), 5.26\left(\mathrm{~s}, 1 \mathrm{H}, \mathrm{H}_{\mathrm{l}}\right), 7.13-$ $7.38\left(\mathrm{~m}, 7 \mathrm{H}, \mathrm{H}_{\mathrm{a}-\mathrm{g}}\right), 7.40-7.43\left(\mathrm{dd}, 2 \mathrm{H}, \mathrm{H}_{\mathrm{mn}}, J=9 \mathrm{~Hz}\right), 8.07-8.10\left(\mathrm{dd}, 2 \mathrm{H}, \mathrm{H}_{\mathrm{pq}}, J=9 \mathrm{~Hz}\right), 8.35$ (s, $\left.1 \mathrm{H}, \mathrm{H}_{\mathrm{h}}\right), 9.09\left(\mathrm{~s}, 1 \mathrm{H}, \mathrm{H}_{\mathrm{k}}\right), 10.15\left(\mathrm{~s}, 1 \mathrm{H}, \mathrm{H}_{\mathrm{o}}\right) ;{ }^{13} \mathrm{C}-\mathrm{NMR}\left(\mathrm{CDCl}_{3}\right): \delta 26.4,41.3,52.6,59.6,103.1$, $103.8,105.1,114.3,115.9,116.7,122.1,127.1,127.7,128,128.3,142.1,143.2,155.4,155.7$, 162.8. Mass $(\mathrm{m} / \mathrm{z})$ : 441. Anal. (\%) for $\mathrm{C}_{24} \mathrm{H}_{19} \mathrm{~N}_{5} \mathrm{O}_{2} \mathrm{~S}$, Calcd. C, 65.29; H, 4.34; N, 15.86; Found: C, 65.30; H, 4.32; N, 15.80 . 
3-Methyl-1-(10H-phenothiazin-2-yl)-4-(2-chlorophenyl)-6-hydroxy-4,5-dihydro-1H-pyrazolo[3,4-d]pyrimidine (3d).Yield 77\%, mp. 186-188 ${ }^{\circ} \mathrm{C}, \mathrm{IR}(\mathrm{KBr}): 3325(\mathrm{NH}), 1610(\mathrm{C}=\mathrm{N})$, $1648(\mathrm{C}-\mathrm{N}), 647$ (C-S-C). ${ }^{1} \mathrm{H}$ NMR $\left(300 \mathrm{MHz}, \mathrm{CDCl}_{3}\right): \delta 2.38\left(\mathrm{~s}, 3 \mathrm{H}, \mathrm{H}_{\mathrm{i}}\right), 5.23\left(\mathrm{~s}, 1 \mathrm{H}, \mathrm{H}_{1}\right), 7.63-$ $7.86\left(\mathrm{~m}, 11 \mathrm{H}, \mathrm{H}_{\mathrm{a}-\mathrm{g}, \mathrm{n}-\mathrm{q}}\right), 8.39\left(\mathrm{~s}, 1 \mathrm{H}, \mathrm{H}_{\mathrm{h}}\right), 9.16\left(\mathrm{~s}, 1 \mathrm{H}, \mathrm{H}_{\mathrm{k}}\right) ;{ }^{13} \mathrm{C}-\mathrm{NMR}\left(\mathrm{CDCl}_{3}\right): \delta 26.1,36.6,52.1$, $60.1,103.1,103.8,104.9,114.2,116.8,122.1,126.7,127.1,127.5,128.2,129,129.6,133.2$, 139.6, 142.1, 143.2, 155.3, 162. Mass (m/z): 460. Anal. (\%) for $\mathrm{C}_{24} \mathrm{H}_{18} \mathrm{~N}_{5} \mathrm{OSCl}$, Calcd. C, 62.67; H, 3.94; N, 15.23; Found: C, 62.65; H, 3.93; N, 15.20.

3-Methyl-1-(10H-phenothiazin-2-yl)-4-(4-chlorophenyl)-6-hydroxy-4,5-dihydro-1H-pyrazolo[3,4-d]pyrimidine (3e). Yield 76\% mp. 153-155 ${ }^{\circ} \mathrm{C}$. IR $(\mathrm{KBr}): 3328(\mathrm{NH}), 1611(\mathrm{C}=\mathrm{N})$, 1647 (C-N), 651 (C-S-C). ${ }^{1} \mathrm{H}$ NMR $\left(300 \mathrm{MHz}, \mathrm{CDCl}_{3}\right): \delta 2.36\left(\mathrm{~s}, 3 \mathrm{H}, \mathrm{H}_{\mathrm{i}}\right), 5.25\left(\mathrm{~s}, 1 \mathrm{H}, \mathrm{H}_{1}\right), 7.32-$ $7.34\left(\mathrm{dd}, 2 \mathrm{H}, \mathrm{H}_{\mathrm{mn}}, J=8.10 \mathrm{~Hz}\right), 7.81-7.84\left(\mathrm{dd}, 2 \mathrm{H}, \mathrm{H}_{\mathrm{pq}} J=7.80 \mathrm{~Hz}\right), 7.88-8.27\left(\mathrm{~m}, 7 \mathrm{H}, \mathrm{H}_{\mathrm{a}-\mathrm{g}}\right.$ ), $8.43\left(\mathrm{~s}, 1 \mathrm{H}, \mathrm{H}_{\mathrm{h}}\right), 9.09\left(\mathrm{~s}, 1 \mathrm{H}, \mathrm{H}_{\mathrm{k}}\right) ;{ }^{13} \mathrm{C}-\mathrm{NMR}\left(\mathrm{CDCl}_{3}\right): \delta 26,41.1,52.1,59.8,103.2,104,105.1$, $114.3,116.4,122.5,127.1,127.7,128,128.4,128.9,131.4,134.4,142.2,143.1,155.4,162.8$. Mass (m/z): 460. Anal. (\%) for $\mathrm{C}_{24} \mathrm{H}_{18} \mathrm{~N}_{5} \mathrm{OSCl}$, Calcd. C, 62.67; H, 3.94; N, 15.23; Found: C, $62.66 ; \mathrm{H}, 3.90 ; \mathrm{N}, 15.25$.

3-Methyl-1-(10H-phenothiazin-2-yl)-4-(2-nitrophenyl)-6-hydroxy-4,5-dihydro-1H-pyrazolo[3,4-d]pyrimidine (3f). Yield 85\%, mp. 213-216 ${ }^{\circ} \mathrm{C}, \mathrm{IR}(\mathrm{KBr}): 3339(\mathrm{NH}), 1605(\mathrm{C}=\mathrm{N})$, 1645 (C-N), 657 (C-S-C). ${ }^{1} \mathrm{H}$ NMR $\left(300 \mathrm{MHz}, \mathrm{CDCl}_{3}\right): \delta 2.47\left(\mathrm{~s}, 3 \mathrm{H}, \mathrm{H}_{\mathrm{i}}\right), 5.18\left(\mathrm{~s}, 1 \mathrm{H}, \mathrm{H}_{\mathrm{l}}\right), 7.25-$ $7.58\left(\mathrm{~m}, 11 \mathrm{H}, \mathrm{H}_{\mathrm{a}-\mathrm{g}, \mathrm{n}-\mathrm{q}}\right), 8.29\left(\mathrm{~s}, 1 \mathrm{H}, \mathrm{H}_{\mathrm{h}}\right), 9.22\left(\mathrm{~s}, 1 \mathrm{H}, \mathrm{H}_{\mathrm{k}}\right) ;{ }^{13} \mathrm{C}-\mathrm{NMR}\left(\mathrm{CDCl}_{3}\right): \delta 26.6,36.3,51.3$, $59.5,103.1,103.7,104.9,114.3,116.8,122,124.8,126.6,127.1,127.8,128,129.1,134,135$, 142.2, 143.2, 148.2, 155.5, 163. Mass (m/z): 470. Anal. (\%) for $\mathrm{C}_{24} \mathrm{H}_{18} \mathrm{~N}_{6} \mathrm{O}_{3} \mathrm{~S}$, Calcd. C, 61.27; H, 3.86; N, 17.86; Found: C, 61.25; H, 3.85; N, 17.84 .

3-Methyl-1-(10H-phenothiazin-2-yl)-4-(3-nitrophenyl)-6-hydroxy-4,5-dihydro-1H-pyrazolo[3,4-d]pyrimidine (3g). Yield 73\%, mp. 189-191 ${ }^{\circ} \mathrm{C}, \mathrm{IR}(\mathrm{KBr}): 3336(\mathrm{NH}), 1612(\mathrm{C}=\mathrm{N})$, 1651 (C-N), 657 (C-S-C). ${ }^{1} \mathrm{H}$ NMR (300 MHz, $\left.\mathrm{CDCl}_{3}\right)$, delta (ppm), 2.33 (s, 3H, Hi), 5.15 (s, $\left.1 \mathrm{H}, \mathrm{H}_{\mathrm{l}}\right), 7.72-7.95\left(\mathrm{~m}, 11 \mathrm{H}, \mathrm{H}_{\mathrm{a}-\mathrm{g}, \mathrm{m}, \mathrm{o}-\mathrm{q}}\right), 8.36\left(\mathrm{~s}, 1 \mathrm{H}, \mathrm{H}_{\mathrm{h}}\right), 9.19\left(\mathrm{~s}, 1 \mathrm{H}, \mathrm{H}_{\mathrm{k}}\right) ;{ }^{13} \mathrm{C}-\mathrm{NMR}\left(\mathrm{CDCl}_{3}\right): \delta$ 26.3, 40.3, 52.6, 59.6, 103.1, 103.7, 105.1, 114.2, 116.2, 121.1, 122.3, 127.1, 127.8, 128, 129.9, 134.1, 140.5, 142.1, 143.5, 147.9, 155.1, 163.2. Mass $(\mathrm{m} / \mathrm{z})$ : 470. Anal. (\%) for $\mathrm{C}_{24} \mathrm{H}_{18} \mathrm{~N}_{6} \mathrm{O}_{3} \mathrm{~S}$, Calcd. C, 61.27; H, 3.86; N, 17.86; Found: C, 61.23; H, 3.83; N, 17.82 .

3-Methyl-1-(10H-phenothiazin-2-yl)-4-(4-methoxyphenyl)-6-hydroxy-4,5-dihydro-1H-pyrazolo[3,4-d]pyrimidine (3h). Yield 73\%, mp. 206-209 ${ }^{\circ} \mathrm{C}$, IR $(\mathrm{KBr}): 3333(\mathrm{NH}), 1616(\mathrm{C}=\mathrm{N})$, 1657 (C-N), 650 (C-S-C). ${ }^{1} \mathrm{H}$ NMR (300 MHz, $\left.\mathrm{CDCl}_{3}\right): \delta 2.37\left(\mathrm{~s}, 3 \mathrm{H}, \mathrm{H}_{\mathrm{i}}\right), 5.28\left(\mathrm{~s}, 1 \mathrm{H}, \mathrm{H}_{\mathrm{l}}\right), 7.31-$ $7.33\left(\mathrm{dd}, 2 \mathrm{H}, \mathrm{H}_{\mathrm{mn}}, J=7.80 \mathrm{~Hz}\right), 7.81-7.84\left(\mathrm{dd}, 2 \mathrm{H}, \mathrm{H}_{\mathrm{pq}}, J=7.80 \mathrm{~Hz}\right), 7.89-7.99\left(\mathrm{~m}, 7 \mathrm{H}, \mathrm{H}_{\mathrm{a}-\mathrm{g}}\right)$, $8.33\left(\mathrm{~s}, 1 \mathrm{H}, \mathrm{H}_{\mathrm{h}}\right), 9.06\left(\mathrm{~s}, 1 \mathrm{H}, \mathrm{H}_{\mathrm{k}}\right) ;{ }^{13} \mathrm{C}-\mathrm{NMR}\left(\mathrm{CDCl}_{3}\right): \delta 26.5,40.1,52.3,60.3,103.1,103.7$, $104.8,114.2,116.3,122.1,126.5,127.9,128.7,129.5,133.2,135.9,142.1,143.1,149.2,155.8$, 163.5. Mass $(\mathrm{m} / \mathrm{z})$ : 455. Anal. (\%) for $\mathrm{C}_{25} \mathrm{H}_{21} \mathrm{~N}_{5} \mathrm{O}_{2} \mathrm{~S}$, Calcd. C, 65.92; H, 4.65; N, 15.37; Found: C, 65.90; H, 4.60; N, 15.33. 


\section{References}

1. El-Said, M. K. Pharmazie 1981, 36, 678.

2. Tilak, S. R.; Tyagi, R.; Goel, B.; Saxena, K. K. Indian drugs 1998, 35, 221.

3. Dominguez, J. N.; Lopez, S.; Charris, J.; Iarruso, L.; Lobo, G.; Semenow, A.; Olson, J. E.; Rosenthal, P. J. J. Med. Chem. 1997, 40, 2726.

4. Lin, G.; Midha, K. K.; Hawes, E. M. J. Heterocycl.Chem. 1991, 28, 215.

5. Raval, J.; Desai, K. K. ARKIVOC 2005, (xiii), 21.

6. Viveros, M.; Amaral, L. Int. J. Antimicrob. Ag. 2001, 17, 225.

7. Amaral, L.; Kristiansen. Int. J. Antimicrob. Ag. 2000, 14, 173.

8. Motohasho, N.; Kurihara, T.; Satoh, K.; Sakagami, H. H.; Mucsi, I.; Pusztai, R.; Szabo, M; Molnar, J. Anticancer Res. 1999, 19, 1837.

9. Motohasho, N.; Kawase, M.; Saito, S.; Sakagami, H. Curr. Drug Targets 2000, 1, 237.

10. Kurihara, T.; Motohasho, N.; Pang, G. L.; Higano, M.; Kiguchi, K.; Molnar, J. Anticancer Res. 1996, 16, 2757.

11. Ghosh, N.; Chattopadhyay, U. In Vivo 1993, 7, 435.

12. Floyd, R. A.; Scheider, J. E.; Zhu, Y. Q.; North, T. W.; Schinazi, F. Proc. Am. Assoc. Cancer. Res. 1993, 34, 359.

13. Kurihara, T.; Motohashi, N.; Sakagami, H. H.; Molnar J. Anticancer Res. 1999, 19, 4081.

14. Kurihara, T.; Nojima, K.; Sakagami, H.; Motohashi, N.; Molnar J. Anticancer Research 1999, 19, 3895.

15. Foroughifar, N.; Mobinikhaledi, A.; Shariatzadeh, S.M.; Masoudnia, M. Asian J. Chem. 2002, 14, 782.

16. Verma, R. S. Green Chem. 1999, 43.

17. Funahashi, K.; Satha, F.; Morita, M.; Noguchi, T. J. Med. Chem. 1989, 32, 2399.

18. Atwal, K. S.; Swanson, B. N.; Unger, S.E.; Floyd, D.M.; Moreland, S.; Hedberg, A.; O'Reilly, B. C. J. Med. Chem. 1991, 34, 806.

19. Kappe, C. O.; Fabian, W. M. F.; Semones, M. A. Tetrahedron 1997, 53, 2803.

20. Xie, W.; Jin, Y.; Wang, P.G. Chemtech 1999, 2, 23.

21. Overman, L. E.; Robinowitz, M. H.; Renhow, P. A. J. Am. Chem. Soc. 1995, 117, 1657.

22. Kappe, C. O.; Falsone, F. S. Synlett 1998, 718.

23. Grover, G. J.; Dzwonczyk, S.; Normadinam, C. S.; Sleph, P.G.; Moreland, S. J. Cardiovasc. Pharmacol. 1995, 28, 289.

24. Kappe, C.O. Tetrahedron 1993, 49, 6937.

25. Ghorba, M. M.; Mohamed, Y. A.; Mohamed, S. A.; Ammar, Y. A. Phosphorus, Sulfur, Silicon 1996, 108, 249.

26. Tsuji, K.; Ishikawa, H. Bioorg. Med .Chem. Lett. 1994, 4, 1601.

27. Kidwai, M.; Mishra, A.D. Bull. Korean Chem. Soc. 2003, 24, 1038.

28. Biginelli, P. Gazz Chim. Ital. 1893, 23, 360.

29. Kappe, C. O.; Rochge, P. J. Heterocycl. Chem. 1989, 26, 55. 
30. Lin, H.; Ding, J.; Chen, X.; Zhang, Z. Molecules 2000, 5, 1240.

31. Foroughifar, N.; Mobinikhaledi; Fathinejad. Phosphorus, Sulfur, Silicon 2003, 178, 495.

32. Sharaf, M. A. F.; Abdel, F. A.; Fattah, A. M.; Khalil, A. M. R. J. Chem. Research (S), 1996, 354.

33. O’Reilly, B. C.; Atwal, K.S. Heterocycles 1987, 26, 1158.

34. Shutalev, A. D.; Kishko, E. A. Sivova, N.; Kuzentsov, A.Y. Molecules 1989, 3, 100.

35. Bhuva, V. R.; Purohit, D. M.; Shah, V. H. International Symposium on Drug Discovery and Process Research 2003, 130, 80,

36. Kappe, C. O. Tetrahedron 1993, 49, 6937. 